\title{
Applying Neural Network to Reconstruction of Phylogenetic Tree
}

\author{
Tujin, Zhu \\ Shenzhen Institute of Advanced Technology, Chinese \\ Academy of Sciences, University of Chinese Academy of \\ Sciences
}

\begin{abstract}
Reconstruction of phylogenetic tree from biological sequences is a fundamental step in molecular biology, but it is computationally exhausting. Our goal is to use neural network to learn the heuristic strategy of phylogenetic tree reconstruction algorithm. We propose an attention model to learn heuristic strategies for constructing circular ordering related to phylogenetic trees. We use alignmentfree K-mer frequency vector representation to represent biological sequences and use unlabeled sequence data sets to train attention model through reinforcement learning. Comparing with traditional methods, our approach is alignment-free and can be easily extended to large-scale data with computational efficiency. With the rapid growth of public biological sequence data, our method provides a potential way to reconstruct phylogenetic tree.
\end{abstract}

\section{CCS CONCEPTS}

- Applied computing $\rightarrow$ Life and medical sciences; Computational biology; Molecular evolution.

\section{KEYWORDS}

Phylogenetic Tree, Attention Model, Reinforcement Learning

\section{ACM Reference Format:}

Tujin, Zhu and Yunpeng, Cai. 2021. Applying Neural Network to Reconstruction of Phylogenetic Tree. In 2021 13th International Conference on Machine Learning and Computing (ICMLC '21), February 26-March 01, 2021, Shenzhen, China. ACM, New York, NY, USA, 7 pages. https://doi.org/10. $1145 / 3457682.3457704$

\section{INTRODUCTION}

Phylogenetic trees reflect the molecular trajectory of living species evolution, thus becomes a fundamental tool in biology. The reconstruction of phylogenetic tree from DNA sequences has been studied in the past several decades. Many methods have been proposed to solve it. The reconstruction of phylogenetic tree has been proved to be NP-complete [1], so how to reconstruct phylogenetic tree is still an open problem in bioinformatics. In this paper, we propose a new phylogenetic tree reconstruction algorithm based on the deep neural network (DNN) and the circular ordering of

Permission to make digital or hard copies of all or part of this work for personal or classroom use is granted without fee provided that copies are not made or distributed for profit or commercial advantage and that copies bear this notice and the full citation on the first page. Copyrights for components of this work owned by others than ACM must be honored. Abstracting with credit is permitted. To copy otherwise, or republish, to post on servers or to redistribute to lists, requires prior specific permission and/or a fee. Request permissions from permissions@acm.org.

ICMLC '21, February 26-March 01, 2021, Shenzhen, China

(C) 2021 Association for Computing Machinery.

ACM ISBN 978-1-4503-8931-0/21/02 . \$15.00

https://doi.org/10.1145/3457682.3457704

\author{
Yunpeng, Cai \\ Shenzhen Institute of Advanced Technology, Chinese \\ Academy of Sciences, University of Chinese Academy of \\ Sciences
}

phylogenetic trees. Our method is a fast method without sequence alignment. Using unannotated biological sequence datasets, we train our model by reinforcement learning.

Phylogenetic tree reconstruction algorithms can be divided into two categories: distance-based and character-based [4]. Both types have two main steps: first, define a criteria to score any tree, and then search the possible tree space to find the optimal tree. Maximum Likelihood methods (ML methods, like FastTree [5] and RAxML [6]) and Bayesian inference methods (like BEAST [8] and MrBayes [7]) are the most popular character-based methods. They all use statistical criteria to score trees. Assuming that each site of biological sequence is independent and identically distributed, they calculate the probability product of all sites as the criteria. Before calculating the criteria, multiple sequence alignment (MSA) must be conducted. Although they yield trees that are easy to interpret, the computations of them are super heavy. Paradoxically, character-based methods depend on MSA, while many MSA methods need phylogenetic tree reconstruction methods to build guide trees (such as MUSCLE [13]). Distance-based methods are based on Minimum Evolution (ME) criteria [12]. they firstly build a distance matrix to represent the genetic distances of all pairs of two biological sequences, and then, construct a tree with minimum tree length. Given a tree topology, Minimum Evolution method use least-squares to calculate the length of all branches and consider the sum of lengths of all branches (tree length) as criteria (called ME criteria). The optimal trees are the trees with minimum tree length. Some approximate methods of ME method run faster and product good phylogenetic trees, like Unweighted Pair-Group Method using Arithmetic Averages (UPGMA) [11] and neighbor-joining (NJ) [10].

The reconstruction of phylogenetic tree can be reduced to combinatorial optimization problem (COP) [1]. Heuristic search is the most popular method to solve COP problems, that the solution space is explored for finding best solutions. Starting from one or a set of initial solutions, it moves to next or next set of candidate solutions step by step until no better solution is found. A specific heuristic policy needs to be artificially designed for each kind of COP. Recently, many learned heuristics using deep neural networks and reinforcement learning are presented to solve COP. Bello et al. [14] and Kool et al. [15] use an attention model to represent heuristics and use Actor-Critic algorithm to train attention model to solve Travelling Salesman Problem (TSP). Dai et al. [16] and Mittal et al. [17] apply Graph Neural Networks (GNN) and Deep Q-Networks [18] to solve large scale COP tasks, like Minimum Vertex Cover and Maximum Cut. Neural networks and reinforcement learning provide a general ideal to solve those NP-hard problems.

In this article, we show the potential of applying machine learning to phylogenetic tree reconstruction by learning a heuristic policy with less artificial design. While the COP problems solved 
by machine learning mentioned above extract information from a known graph, phylogenetic tree reconstruction recovers a tree-like graph from DNA or protein sequences data. It is harder to let neural networks learn to construct graph and output a graph with complex structure. So, we train neural networks to output circular orderings (defined in next section) related to phylogenetic trees, and then convert circular orderings to phylogenetic trees. We will introduce the concept of phylogenetic tree, related circular orderings and the detail of model and training method we use. Finally, we show that our method is better than some popular distance-based methods on small-scaled tasks.

\section{PRELIMINARIES OF TREE}

\subsection{Tree concepts}

A phylogenetic tree is a graph $G$ without loops as a set of nodes $V(G)$ and a set of edges $E(G)$. each edge represents the evolutional distance between two nodes, and each terminal node (leaf) represents a species or gene, each interior node represents an unknown ancestry. A rooted tree has a specified node, called root. The root node is the ancestry of all other nodes on the same tree, while the root of unrooted tree is unknown. The number of edges connected to a node $v$ is the degree $d(v)$ of $v$. If the root is known, the degree of root node is 2 , and all non-root interior node have degree of 3 . Such tree is called binary tree or bifurcating tree. An unrooted binary tree with $n$ leaves has $2 n-3$ edges and $n-2$ interior nodes.

A distance map $\delta$ is a function: $X \times X \rightarrow \mathbb{R}$. $\delta$ satisfies three conditions: $\delta(x, y)=\delta(y, x), \delta(x, x)=0$ and $\delta(x, y) \leq \delta(x, z)+$ $\delta(z, y)$ for $\forall x, y, z \in X$. Such distance map is also called tree metric.

There are many distance-based methods like NJ [10], UPGMA [11] and ME method [12] have been developed to construct phylogenetic tree, which based on distance matrix calculated from a tree metric $\delta$, Those methods base on an assumption that the distance between any pair of two sequences is equal to the total length of path connecting two sequences in true phylogenetic tree:

$$
\delta(x, y)=\sum_{e \in P(x, y)} w(e) \forall x, y \in X
$$

where $P(x, y)$ represents the set of edges in the path from leaf $x$ to leaf $y$ on the tree, $w(e)$ represents a nonnegative real-valued weighting (length) of edge $e$.

Calculating from a tree metric $\delta$, a binary X-tree is a binary phylogenetic tree that satisfied above equation 1 , with leaves set $X$, and $|X| \geq 3[3,9,19]$.

Let $T$ be a binary X-tree with leaf set $X$, we denote $\Delta_{y, z}^{x}$ as the distance from leaf $x$ to the path $P(x, y)$ :

$$
\Delta_{y, z}^{x}=\frac{1}{2}(\delta(x, y)+\delta(x, z)-\delta(y, z)) \forall x, y, z \in X
$$

Let $B$ be a subset of $X$, which is not equal to $X$ and not empty. We denote the set $\{B, X-B\}$ as a bipartition of $X$. If we cut an edge in a binary X-tree $T$ with $n$ leaves, we get a unique bipartition corresponded to the edge. We denote $\Sigma(T)$ as the set of all possible bipartitions generated from $T$ by cutting an edge. The size of $\Sigma(T)$ is equal to the number of edges of $T:|\Sigma(T)|=2 n-3$. Two binary Xtree $T_{1}$ and $T_{2}$ are considered equivalent if and only if $\Sigma\left(T_{1}\right)=\Sigma\left(T_{2}\right)$ [3].
Let $\pi=\left(x_{1}, x_{2}, \ldots, x_{n}\right)$ be a circular permutation (the next of $x_{n}$ is $x_{1}$ ) of $X$ and denote $\Sigma^{\circ}(\pi)$ as the set of all bipartitions generated by separating the circular permutation in a plane using a line segment. Obviously, the size of $\Sigma^{\circ}(\pi)$ is $\left(\begin{array}{l}n \\ 2\end{array}\right)$.

\subsection{Circular ordering}

The definition of circular ordering is as follows. Considering a binary X-tree $T$ and a circular permutation $\pi$ of $X, \pi$ is regarded as circular ordering of $T$ if

$$
\Sigma(T) \subseteq \Sigma^{\circ}(\pi)
$$

There are some useful properties of circular ordering and binary X-tree proved by Semple and Steel [3]. Given a circular permutation $\pi$ of leaf set $X$, and $|X|=|\pi|=n \geq 3$, the number of possible nonequivalent binary $X$-tree is $\frac{1}{n-1}\left(\begin{array}{c}2 n-4 \\ n-2\end{array}\right)$. Given $n$ leaves, the number of possible unrooted binary X-trees is $\frac{(2 n-5) !}{2^{n-3}(n-3) !}$. The later number is much larger than the previous one. If we know the precise circular ordering of the input biological sequences, the search space can be significantly reduced. One way to reconstruct a phylogenetic tree is that construct circular ordering $\pi$ of leaf set $X$, and then, reconstruct tree $T$ from $\pi$.

Minimum Evolution criteria (tree length) can be used to score circular ordering of a given sequence set $X$. Let $T$ be a binary X-tree with leaf set $X, \pi=\left(x_{1}, x_{2}, \ldots, x_{n}\right)$ be a circular ordering of $X$ and $\delta$ be the corresponding tree metric. a circular path tuple of $\pi$ is a tuple of $\left(p_{1}, p_{2}, \ldots, p_{n}\right)$, where $p_{i}=P\left(x_{i}, x_{i+1}\right)$ is the path from $x_{i}$ to $x_{i+1}$ in the tree $T$ (note that $x_{n+1}=x_{1}$ ). Each edge in the tree $T$ occurs in exactly two of the paths in circular path tuple $[3,19]$, so that, the tree length $L(T)$ can be calculated as follows:

$$
L(T)=\frac{1}{2} L(\pi)=\frac{1}{2} \sum_{i=1 \text { to } n} \delta\left(x_{i}, x_{i+1}\right)
$$

where $x_{n+1}=x_{1}$.

There exists an Algorithm which can convert circular ordering into phylogenetic tree [25]. The remaining question is how to construct circular ordering from input sequence set $X$ and tree metric $\delta$. We propose a neural network model to construct circular ordering from $X$ and $\delta$.

\section{METHOD}

We divide our method of the reconstruction of phylogenetic tree into two steps. Step one: circular orderings is inferred by an attention model using input sequences and a tree metric. Step two: a binary X-tree is reconstructed by a stepwise addition algorithm from the circular ordering [25]. The overall structure of our approach is showed in Figure 1. We will describe in detail about our approach in this section.

\subsection{K-mer representation and tree metric}

Biological sequence is usually represented as a string of variable length, which cannot be treated directly by most of machine learning model. We convert sequence $s$ into a K-mer frequency vector $x$ by mapping it to a $4^{k}$ dimensions space. A K-mer is a sequence of length $k$, and the number of all possible K-mers of DNA sequence is 


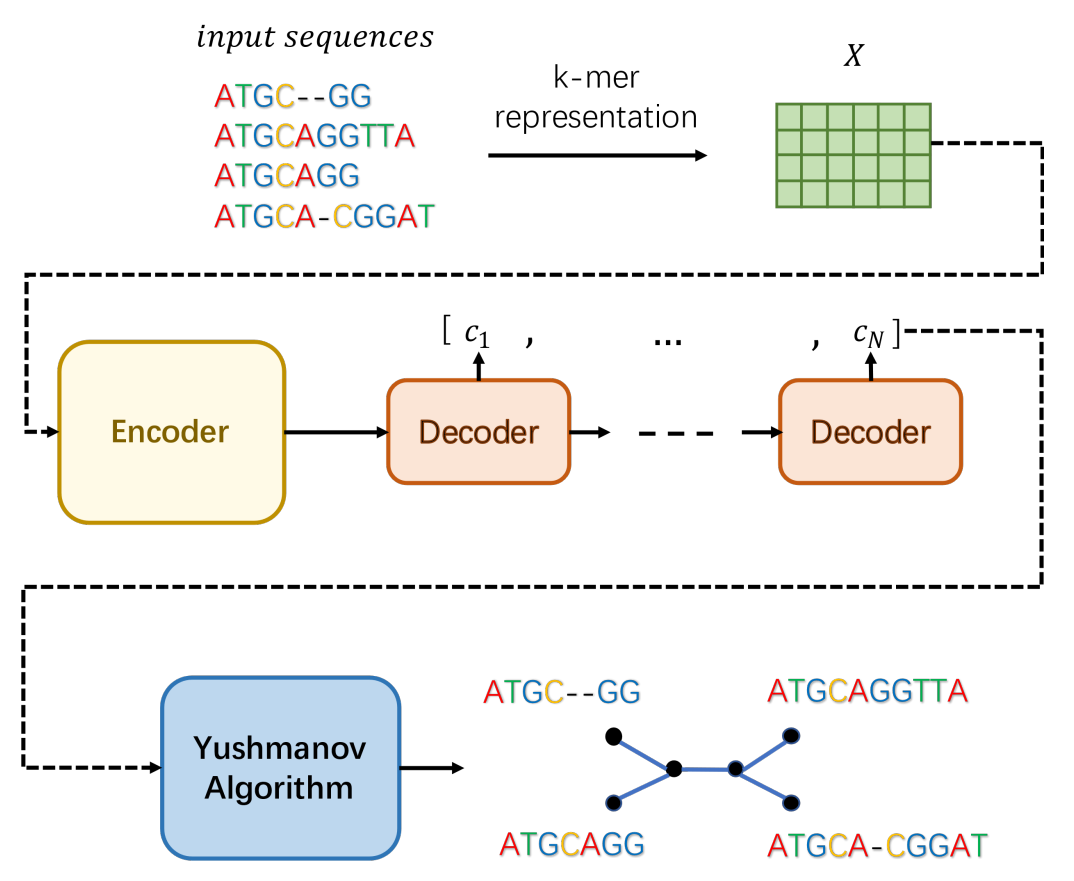

Figure 1: Overall structure of our phylogenetic tree reconstruction method.

$4^{k}$ ( 4 is the size of alphabet set of nucleotides). A K-mer frequency vector $x$ of a sequence $s$ is a vector of size of $4^{k}$. Each element of the vector indicates the proportion of the number a K-mer in the total number of K-mer in a sequence.

A derivable tree metric of biological sequences is needed to optimize neural network model. We use Euclidean distance between each pair of K-mer frequency vector as tree metric to measure the evolutionary distance of corresponding sequences. Euclidian distance between two K-mer frequency vectors is calculated as follows:

$$
\delta_{E}\left(x_{1}, x_{2}\right)=\left(\sum_{i}\left(x_{1}^{i}-x_{2}^{i}\right)^{2}\right)^{\frac{1}{2}}
$$

where $x_{1}^{i}$ and $x_{2}^{i}$ respectively represent the $i^{\text {th }}$ value of vector $x_{1}$ and vector $x_{2}$.

\subsection{Attention model}

In the following, we describe the attention model that takes K-mer frequency matrix $X$ of sequences as input and output a circular ordering of the row of matrix $X$. Like other neural sequence transduction models [20], the attention model we use consists of an encoder and a decoder. This model is similar to the model provided by Kool et al. [15]. Given a biological sequence (DNA or protein sequence) set $S$ of size $N$, we convert $S$ into K-mer frequency ma$\operatorname{trix} X \in \mathbb{R}^{N \times d_{x}}$. Each row vector in $X$ is a K-mer frequency vector representing a sequence in $S$. The dimension of K-mer frequency vector is: $d_{x}=4^{k}$ (for DNA).
3.2.1 Attention mechanism. The key component of attention model is a special attention mechanism called scaled dot-product attention [21]. Scaled dot-product attention of task-related queries is calculated as follows:

$$
\operatorname{att}(Q, K, V)=\operatorname{softmax}\left(\frac{Q K^{T}}{\sqrt{d_{k}}}\right) V
$$

where $Q \in \mathbb{R}^{N \times d_{q}}, K \in \mathbb{R}^{N \times d_{k}}$ and $V \in \mathbb{R}^{N \times d_{v}}$ represent the concatenated matrix of query, key and value vectors respectively, and $N$ is the size of input samples. The attention with $Q=K=V$ is called Self-Attention.

Multi-Head Attention (MHA) is presented by Vaswani, which can get more information than single head attention [21]. MHA perform multiple attention heads to get multiple information of different perspective.

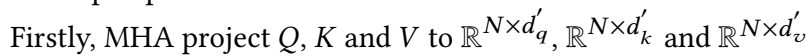
space using different parameters $W^{Q}, W^{K}$ and $W^{V}$ respectively for $M$ times. The projection dimension of $Q, K$ and $V$ are $d_{q}^{\prime}=$ $d_{q} / M, d_{k}^{\prime}=d_{k} / M$ and $d_{v}^{\prime}=d_{v} / M$. Then, MHA calculates each attention of query matrix $Q^{(m)}$ to $K^{(m)}$ and $V^{(m)}$ using equation 6 . Finally, the attentions of all heads are concatenated to a single matrix by projecting each attention back to $\mathbb{R}^{N \times d_{v}}$ using parameter $W_{m}^{O} \in \mathbb{R}^{d_{v}^{\prime} \times d_{v}}:$

$$
\operatorname{MHA}(Q, K, V)=\sum_{m=1}^{M} \operatorname{att}\left(Q W_{m}^{Q}, K W_{m}^{K}, V W_{m}^{V}\right) W_{m}^{O}
$$

Multi-Head Self-Attention (MHSA) is a special MHA with $Q=K=$ $V: M H S A(H)=M H A(H, H, H)$. 


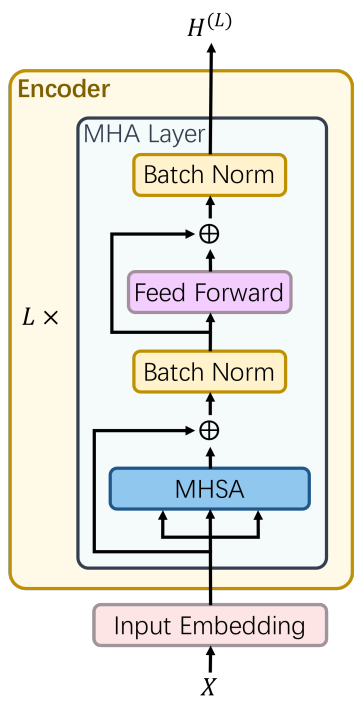

Figure 2: The architecture of encoder

3.2.2 Encoder. The encoder we use is showed in Figure 2. The structure of encoder is inspired by Kool et al. [15]. Before calculating encoder, we calculate an embedding matrix $H^{(0)} \in \mathbb{R}^{N \times d_{h}}$ of input matrix $X \in \mathbb{R}^{N \times d_{x}}$ by a Linear layer with parameters $W^{x} \in R^{d_{x} \times d_{h}}$ and $b^{x} \in R^{N}$ :

$$
H^{(0)}=X W^{x}+b^{x}
$$

The encoder consists of $L$ MHA layers, and each MHA layer has a Multi-Head Self-Attention sublayer, followed by a full-connected Feed-Forward sublayer. The Feed-Forward sublayer (FF) has only one hidden layer and uses ReLu as activation function. Skipconnection [22] and batch normalization (BN) [23] are added to both sublayers. The output of final layer of encoder is denoted as $H^{(L)} \in \mathbb{R}^{N \times d_{h}}$. The output $H^{(l)}$ of each MHA layer is calculated as follows:

$$
\begin{gathered}
\hat{H}^{(l)}=B N^{(l)}\left(H^{(l-1)}+\operatorname{MHSA}\left(H^{(l-1)}\right)\right) \\
H^{(l)}=B N^{(l)}\left(\hat{H}^{(l)}+F F^{(l)}\left(\hat{H}^{(l)}\right)\right)
\end{gathered}
$$

where $B N^{(l)}$ is a learnable batch normalization layer.

The self-attention of $H^{(l)}$ of encoder can be calculated in parallel. The output of Encoder $H^{(L)}$ will be the input of decoder.

3.2.3 Decoder. The architecture of decoder is show in Figure 3. Decoder output a circular ordering $\pi_{\text {index }}=\left(c_{1}, c_{2} \ldots, c_{N}\right)$ of row vector in matrix $X$. We define the ordered sequence of all selected row as the state of decoder at time $t$ : $_{\text {state }}=\left(c_{1}, c_{2}, \ldots, c_{t}\right)$, and state $_{0}$ is an empty sequence. Decoder unit output a probability distribution of row vectors. According to the probability distribution, an unvisited row will be selected (random sampling for training, greedy selecting for tree reconstruction) as $c_{t}$ to form a new state state $_{t}$. Because the computation of decoder unit at step $t$ is related to the state of previous step, decoder unit must be computed sequentially.

There are one masked Multi-Head Attention (masked MHA) sublayer and one attention distribution sublayer in Decoder unit. The

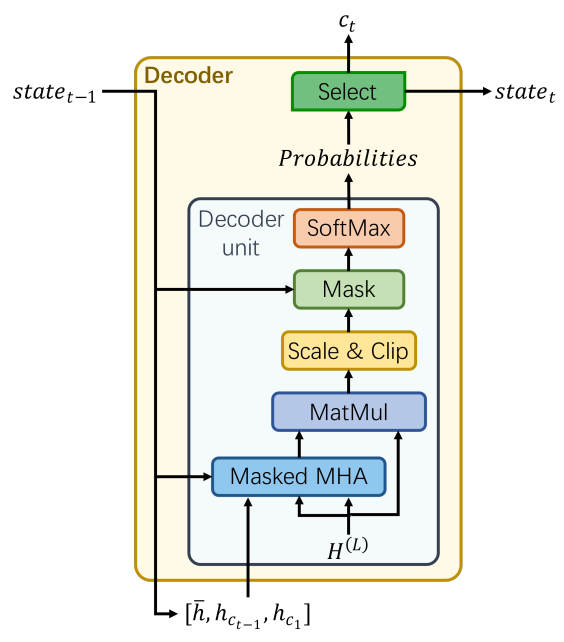

Figure 3: The architecture of decoder

masked MHA is similar to the MHA mentioned above. The query matrix of masked MHA at step $t$ is the aggregate information of current state:

$$
Q_{t}=\left[\bar{h}, h_{c_{t-1}}, h_{c_{1}}\right] \in \mathbb{R}^{1 \times 3 d_{h}}
$$

The $h_{i}$ is the vector of $i^{t h}$ row in $H^{(L)}$ and $\bar{h}$ is the aggregate information of $H^{(L)}$ :

$$
\bar{h}=\frac{1}{N} \sum_{i=1}^{N} H_{i}^{(L)}
$$

The output of encoder is the key matrix and value matrix of masked MHA for each step: $K_{t}=V_{t}=H^{(L)}$. The output $\hat{H}_{t} \in \mathbb{R}^{1 \times d_{h}}$ of masked MHA is calculated as:

$$
\hat{H}_{t}=M M H A\left(Q_{t}, H^{(L)}, H^{(L)}\right)
$$

A mask operation is added to the masked MHA when the probability distribution is calculated to prevent from selecting a visited row.

The attention distribution sublayer of decoder unit is a special scaled dot-product attention, which output the probability distribution of rows of $H^{(L)}$. It is calculated as:

$$
\operatorname{Pr}_{t}=\operatorname{softmax}\left(\frac{\hat{H}_{t}\left(H^{(L)}\right)^{T}}{\sqrt{d_{h}}}\right)
$$

with the same mask operation as masked MHA.

\subsection{Training method}

We use a reinforcement learning algorithm called REINFORCE with baseline [15] to train the model above. Given an input matrix $X$ representing $N$ DNA sequences, the output of model is $\pi=$ $\left(c_{1}, c_{2}, \ldots, c_{N}\right)$. We define the tree length (see equation 4$)$ as the reward of $\pi$ :

$$
L(\pi \mid X)=\frac{1}{2} \sum_{i=1}^{N} \delta_{E}\left(x_{c_{i}}, x_{c_{i+1}}\right)
$$

where $c_{N+1}=c_{1}$. The loss of model is:

$$
\mathcal{L}(\theta)=\mathbb{E}_{X}[(L(\pi \mid X)-b(X)) \cdot \ln (\operatorname{Pr}(\pi \mid X ; \theta))]
$$


$\operatorname{Pr}(\pi \mid X ; \theta)$ is the probability of $\pi=\left(c_{1}, c_{2}, \ldots, c_{N}\right)$ :

$$
\operatorname{Pr}(\pi \mid X ; \theta)=\prod_{t=1}^{N} \operatorname{Pr}_{t}
$$

Baseline $b(X)$ is the reward of the best model currently, and it will be updated in each epoch of training. We use Adam [24] to optimize the model.

\subsection{Reconstructing phylogenetic tree from circular ordering}

Yushmanov provides an effective and efficient algorithm that reconstruct a X-tree from a K-mer frequency matrix $X$ and a circular ordering $\pi[9,25]$. We call it Yushmanov algorithm (see Algorithm 1).

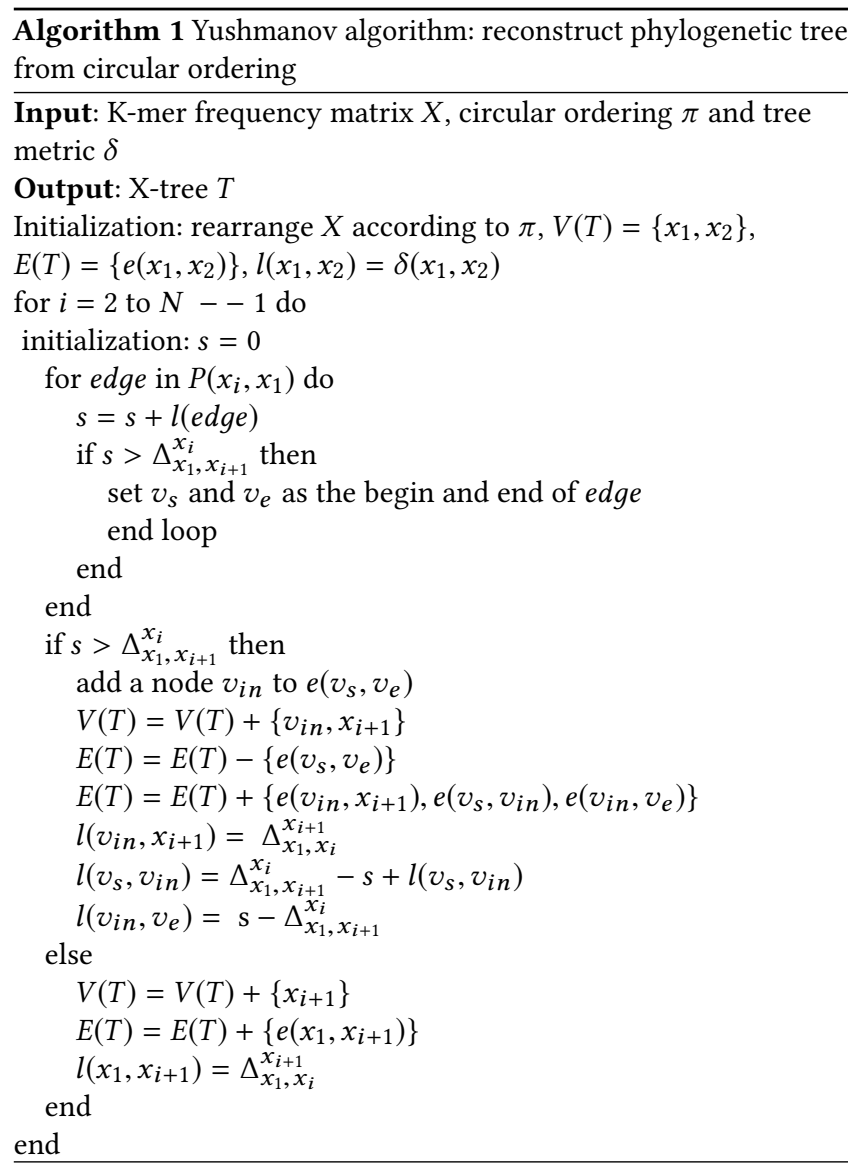

We make some change on Yushmanov algorithm. We firstly rearrange $X$ by the circular ordering of its rows, $\pi_{\text {index }}=$ $\left(c_{1}, c_{2}, \ldots, c_{N}\right)$, given by attention model. In $i^{t h}$ step of algorithm, each edge in the path between $x_{i}$ and $x_{1}$ is checked to find best edge to join $x_{i+1}$. Let $v_{s}$ and $v_{e}$ are the start node and end node of the target edge to join $x_{i+1}$. When leaf $x_{i+1}$ is join to the target edge, a new interior node $v_{i n}$ connected to leaf $x_{i+1}$ will be added on the target edge, now divided into edge $e\left(v_{s}, v_{i n}\right)$ and $e\left(v_{i n}, v_{e}\right)$. The length of three edges $\left(e\left(v_{s}, v_{i n}\right), e\left(v_{i n}, v_{e}\right)\right.$ and a new edge $\left.e\left(v_{i n}, x_{i+1}\right)\right)$ need to be updated. The complexity of this algorithm is $O\left(N^{2}\right)$.

\section{EXPERIMENT}

We test our model on the tasks of reconstruction of phylogenetic tree with $N=15$. The dataset we use is from GreenGenes database [26]. The dataset contains 331550 aligned full-length 16s rRNA sequence, which is available on the homepage of FastTree [5]. We divide randomly raw dataset into training set (330050 16s rRNA sequences) and test set (1500 16s rRNA sequences). We also randomly divide test set into 100 reconstruction tasks of phylogenetic tree of 15 sequences.

We set $k=6$ for K-mer representation, when convert 16s rRNA sequence into K-mer frequency vector. We set $M=8$ for MHA. There are two key hyper parameters: the embedding dimension $d_{h}$ of the input embedding module and the number $L$ of MHA layers of Encoder. We try all combinations of $d_{h} \in\{128,256,512\}$ and $L \in\{2,3,4,5\}$. The model is trained for 100 epochs. In each epoch, $12800 * 15$ sequences are randomly selected from training set to form 12800 reconstruction tasks of phylogenetic tree of 15 sequences. We run batch training with batch size of 512 in each epoch. Before training, a validation set of 5000 reconstruction tasks is selected randomly from train set.

Figure 4 shows the average tree length on validation set of as a function of the number of training batches during training. Except for the condition of $d_{h}=512$ and $L=4$ or 5 , average tree length converges to the minimum value. In distance-based method of reconstruction of phylogenetic tree, what we want is the tree with minimum tree length (Minimum Evolution criteria). The result of parameter of $d_{h}=128$ and $L=2$ is effective enough for subsequent task of reconstruction of tree.

In order to show the accuracy of inferred tree, a reference tree is estimated as true evolutionary tree for each reconstruction task of phylogenetic tree in test set using RAxML [6] of version 8.2.12. We conduct a rapid bootstrap analysis of 1000 replicates to generate 1000 bootstrap maximum likelihood trees, then a majority-rule consensus tree [2] is built from 1000 bootstrap maximum likelihood trees as the reference tree for 16s rRNA sequences.

We compared our method with different hyper parameters to neighbor-joining (NJ), Unweighted Pair-Group Method using Arithmetic Averages (UPGMA) and Minimum Evolution method using least-squares (ME) on test set. Normalized Robinson-Foulds distance (nRF) [27] is used to measure the dissimilarity between two trees. The nRF distance ranges from 0 (two trees are identical) to 1 (two trees are completely different), which is calculated by dendroPy library [28]. We also calculate the logarithm likelihood of trees in evolutionary model [2], the tree with larger likelihood is a better estimate of true tree. Table 1 show the average nRF distances from inferred trees to reference trees and average logarithm likelihood of the trees, calculated by our method and other methods, on test set of 100 reconstruction tasks. Theoretically, the Minimum Evolution method is the most accurate one in the distance method. Other methods, including our method, are approximation algorithm of the minimum evolution method. However, Minimum Evolution method is time-consuming, it will reduce the number of iterations in application. So, the difference between the $\mathrm{NJ}$ trees and ME trees is small in practice. Our method works well when the parameter is $d_{h}=512, L=2$, and it performs better than one of the traditional distance-based method UPGMA. Comparing with existing methods, 

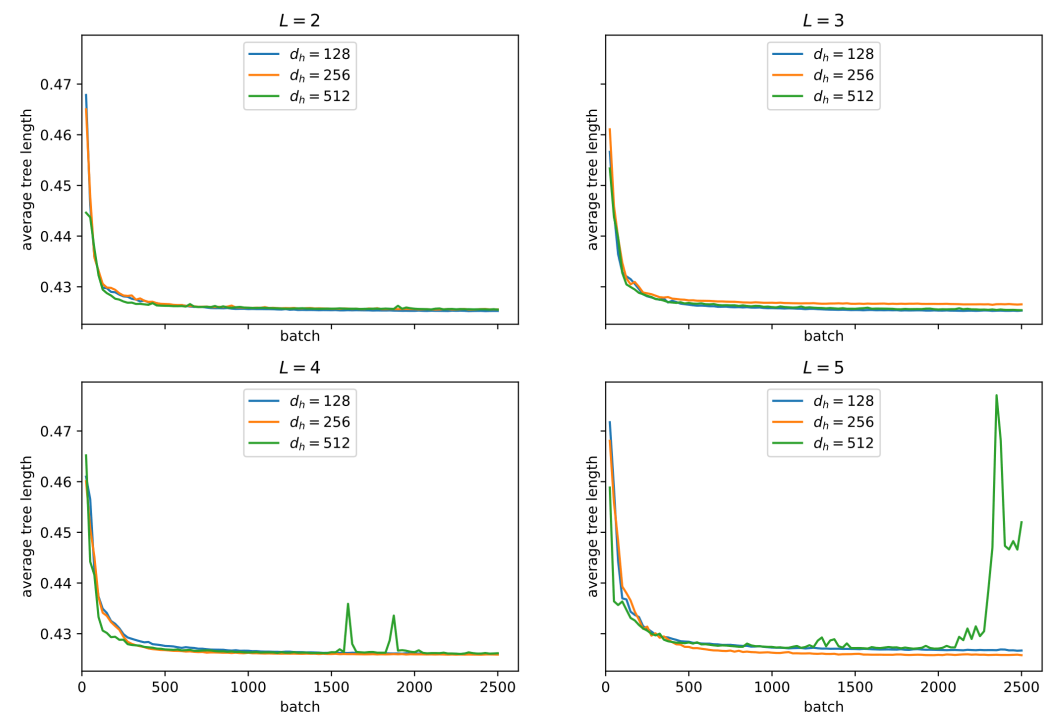

Figure 4: Average tree length on validation as a function of the number of training batches

Table 1: nRF distance to reference tree and logarithm likelihood in evolutionary model

\begin{tabular}{llll}
\hline Method & & Average nRF distance & Average logarithm likelihood \\
\hline \hline Our method & $d_{h}=128, L=2$ & 0.298 & -7495.580 \\
& $d_{h}=128, L=3$ & 0.307 & -7491.102 \\
& $d_{h}=128, L=4$ & 0.335 & -7499.044 \\
& $d_{h}=128, L=5$ & 0.627 & -7738.095 \\
& $d_{h}=256, L=2$ & 0.308 & -7487.377 \\
& $d_{h}=256, L=3$ & 0.313 & -7494.755 \\
& $d_{h}=256, L=4$ & 0.341 & -7500.299 \\
& $d_{h}=256, L=5$ & 0.364 & -7508.352 \\
& $d_{h}=512, L=2$ & $\mathbf{0 . 2 9 7}$ & -7480.490 \\
NJ & $d_{h}=512, L=3$ & 0.322 & -7487.891 \\
UPGMA & $d_{h}=512, L=4$ & 0.343 & -7495.685 \\
ME & $d_{h}=512, L=5$ & 0.751 & -7881.783 \\
& & 0.256 & -7468.533 \\
& & & \\
\hline
\end{tabular}

our approach is alignment-free and can be easily extended to largescale data with computational efficiency. It proves that machine learning has the ability to learn the heuristics for reconstruction of phylogenetic tree on 16s rRNA sequences.

\section{CONCLUSION}

In this article we propose a new phylogenetic tree reconstruction method that construct a circular ordering by a learned neural network (attention model) from biological sequences and reconstruct phylogenetic tree from circular ordering using a $O\left(n^{2}\right)$ algorithm. We consider our approach as a meaningful attempt to reconstruct phylogenetic tree by learning heuristics, while the reconstruction of tree is still full of controversy. Training attention model by reinforcement learning, we can apply this method to any homologous genes, requiring only a large dataset of genes, which is easily obtained from public database. Our method is alignment-free, so it can be even applied to reconstruction of phylogenetic tree on whole genome sequences if we increase the size of K-mer to fit the size of 
genome sequences. Compared to another distance-based method UPGMA, our method constructs a better phylogenetic tree. The most key shortage of our approach is that it is not a complete endto-end method for reconstruction of phylogenetic tree, because the solution of reconstruction is not a sequential decision process. In the future research, graph neural networks may be the key technology to reconstruct tree end-to-end using machine learning. With the development of next generation sequencing technology, another challenge is to apply machine learning method to reconstructions of large-scale phylogenetic tree after our first attempt on small-scale phylogenetic tree.

\section{ACKNOWLEDGMENTS}

We thank the anonymous reviewers for their constructive comments. This work is supported by the Shenzhen Science and Technology Program (Grant No. JCYJ20180507182818013 and No. JCYJ20180703145002040) and the Strategic Priority Research Program of Chinese Academy of Sciences (Grant No. XDB 38050100).

\section{REFERENCES}

[1] Foulds, L. R., \& Graham, R. L. (1982). The steiner problem in phylogeny is NPcomplete. Advances in Applied Mathematics, 3(1). https://doi.org/10.1016/S01968858(82)80004-3

[2] Yang, Z. (2014). Molecular evolution: a statistical approach. Oxford University Press.

[3] Semple, C., \& Steel, M. (2004). Circular permutations and evolutionary trees. Advances in Applied Mathematics, 32(4). https://doi.org/10.1016/S0196-8858(03) 00098-8

[4] Yang, Z., \& Rannala, B. (2012). Molecular phylogenetics: Principles and practice In Nature Reviews Genetics (Vol. 13, Issue 5). https://doi.org/10.1038/nrg3186

[5] Price, M. N., Dehal, P. S., \& Arkin, A. P. (2010). FastTree 2 - Approximately maximum-likelihood trees for large alignments. PLoS ONE, 5(3). https://doi.org/ 10.1371/journal.pone.0009490

[6] Stamatakis, A. (2014). RAxML version 8: A tool for phylogenetic analysis and post-analysis of large phylogenies. Bioinformatics, 30(9). https://doi.org/10.1093/ bioinformatics/btu033

[7] Ronquist, F., Teslenko, M., Van Der Mark, P., Ayres, D. L., Darling, A., Höhna S., Larget, B., Liu, L., Suchard, M. A., \& Huelsenbeck, J. P. (2012). Mrbayes 3.2: Efficient bayesian phylogenetic inference and model choice across a large model space. Systematic Biology, 61(3). https://doi.org/10.1093/sysbio/sys029

[8] Bouckaert, R., Heled, J., Kühnert, D., Vaughan, T., Wu, C. H., Xie, D., Suchard, M. A., Rambaut, A., \& Drummond, A. J. (2014). BEAST 2: A Software Platform for Bayesian Evolutionary Analysis. PLoS Computational Biology, 10(4). https: //doi.org/10.1371/journal.pcbi.1003537

[9] Makarenkov, V., \& Leclerc, B. (1997). Circular orders of tree metrics, and their uses for the reconstruction and fitting of phylogenetic trees. https://doi.org/10. 1090/dimacs/037/11
[10] Saitou, N., \& Nei, M. (1987). The neighbor-joining method: a new method for reconstructing phylogenetic trees. Molecular Biology and Evolution, 4(4). https: //doi.org/10.1093/oxfordjournals.molbev.a040454

[11] Sneath, P. H. A., \& Sokal, R. R. (1973). Unweighted pair group method with arithmetic mean. Numerical Taxonomy, 230-234.

[12] Desper, R., \& Gascuel, O. (2005). The minimum evolution distance-based approach to phylogenetic inference. Mathematics of Evolution and Phylogeny, 1.

[13] Edgar, R. C. (2004). MUSCLE: Multiple sequence alignment with high accuracy and high throughput. Nucleic Acids Research, 32(5). https://doi.org/10.1093/nar/ gkh340

[14] Bello, I., Pham, H., Le, O. V., Norouzi, M., \& Bengio, S. (2019). Neural combinatorial optimization with reinforcement learning. 5th International Conference on Learning Representations, ICLR 2017 - Workshop Track Proceedings.

[15] Kool, W., Van Hoof, H., \& Welling, M. (2019). Attention, learn to solve routing problems! 7th International Conference on Learning Representations, ICLR 2019.

[16] Dai, H., Khalil, E. B., Zhang, Y., Dilkina, B., \& Song, L. (2017). Learning combinatorial optimization algorithms over graphs. Advances in Neural Information Processing Systems, 2017-December.

[17] Mittal, A., Dhawan, A., Manchanda, S., Medya, S., Ranu, S., \& Singh, A. (2019). Learning heuristics over large graphs via deep reinforcement learning. arXiv preprint arXiv:1903.03332.

[18] Mnih, V., Kavukcuoglu, K., Silver, D., Graves, A., Antonoglou, I., Wierstra, D., \& Riedmiller, M. (2013). Playing atari with deep reinforcement learning. arXiv preprint arXiv: 1312.5602

[19] Korostensky, C., \& Gonnet, G. H. (2000). Using traveling salesman problem algorithms for evolutionary tree construction. Bioinformatics, 16(7). https://doi. org/10.1093/bioinformatics/16.7.619

[20] Cho, K., Van Merriënboer, B., Gulcehre, C., Bahdanau, D., Bougares, F., Schwenk, H., \& Bengio, Y. (2014). Learning phrase representations using RNN encoderdecoder for statistical machine translation. EMNLP 2014 - 2014 Conference on Empirical Methods in Natural Language Processing, Proceedings of the Conference. https://doi.org/10.3115/v1/d14-1179

[21] Vaswani, A., Shazeer, N., Parmar, N., Uszkoreit, J., Jones, L., Gomez, A. N., Kaiser, Ł., \& Polosukhin, I. (2017). Attention is all you need. Advances in Neural Information Processing Systems, 2017-December.

[22] He, K., Zhang, X., Ren, S., \& Sun, J. (2016). Deep residual learning for image recognition. Proceedings of the IEEE Computer Society Conference on Computer Vision and Pattern Recognition, 2016-December. https://doi.org/10.1109/CVPR. 2016.90

[23] Ioffe, S., \& Szegedy, C. (2015). Batch normalization: Accelerating deep network training by reducing internal covariate shift. 32nd International Conference on Machine Learning, ICML 2015, 1.

[24] Kingma, D. P., \& Ba, J. L. (2015). Adam: A method for stochastic optimization. 3rd International Conference on Learning Representations, ICLR 2015 - Conference Track Proceedings.

[25] Yushmanov, S. V. (1984). Construction of a tree with p leaves from $2 p-3$ elements of its distance matrix. Matematicheskie Zametki, 35, 877-887.

[26] DeSantis, T. Z., Hugenholtz, P., Larsen, N., Rojas, M., Brodie, E. L., Keller, K., Huber, T., Dalevi, D., Hu, P., \& Andersen, G. L. (2006). Greengenes, a chimerachecked 16S rRNA gene database and workbench compatible with ARB. Applied and Environmental Microbiology, 72(7). https://doi.org/10.1128/AEM.03006-05

[27] Robinson, D. F., \& Foulds, L. R. (1981). Comparison of phylogenetic trees. Mathematical biosciences, 53(1-2), 131-147.

[28] Sukumaran, J., \& Holder, M. T. (2010). DendroPy: A Python library for phylogenetic computing. Bioinformatics, 26(12). https://doi.org/10.1093/bioinformatics/ btq228 